\title{
Cost-effectiveness in the diagnosis of sarcoidosis: the conjunctival biopsy
}

\section{Abstract}

Purpose Sarcoidosis is a multi-system disease that often affects ocular structures. The definitive diagnosis of sarcoidosis requires tissue biopsy for confirmation. Conjunctival biopsy is a simple and relatively inexpensive diagnostic tool. This study was undertaken to determine the utility and cost of conjunctival biopsy for the diagnosis of sarcoidosis compared with other diagnostic biopsy sites. Methods A retrospective study was performed of all conjunctival biopsies taken for sarcoidosis at the Mayo Clinic Rochester between 1982 and 1995. All case histories were reviewed. Data regarding biopsy of tissues confirming sarcoidosis was collected. The costs of performing and evaluating all biopsy techniques were obtained from the estimating department.

Results Forty-one cases of sarcoidosis were identified that had undergone conjunctival biopsies. Twenty-one patients had a positive conjunctival biopsy for sarcoidosis, and 20 patients had a negative conjunctival biopsy but a positive biopsy of another organ. In all positive biopsies, special stains for acid fast organisms and fungi were negative.

Conclusions Conjunctival biopsy is a simple, relatively inexpensive procedure that may be helpful in establishing the diagnosis of sarcoidosis with a negligible complication rate. Positive results are similar for conjunctival biopsy and mediastinoscopy. About 10 patients can be evaluated with conjunctival biopsy for the cost of one patient undergoing mediastinoscopy. Multiple levels of the tissue must be examined, as distribution of the granulomas may be random. Also, bilateral conjunctival specimens should be obtained to increase the positive yield.

Key words Biopsy, Conjunctiva, Sarcoidosis

Sarcoidosis is a systemic disease characterised by granulomatous infiltration of a variety of tissues. Diagnosis of the disease begins with the clinical suspicion, supplemented by radiological and laboratory data. ${ }^{1}$ Clinicopathological correlation is essential and the presence of noncaseating granulomas with negative staining for acid fast organisms and fungi is strongly suggestive of the diagnosis in the appropriate clinical setting. Biopsy is an important tool in establishing the diagnosis.

Pulmonary involvement is the most common clinical manifestation of the disease.

Transbronchial and mediastinal biopsy can be used to establish the diagnosis of sarcoidosis by directing the biopsy to clinically involved tissue. Bronchoalveolar lavage can contribute data supportive of sarcoidosis without a specific tissue diagnosis. $^{2}$

Involvement of the eye in sarcoidosis is common. $^{3-6}$ The most common ophthalmological manifestation is anterior segment granulomatous infiltration.

Conjunctival biopsy has been described in the literature since the 1950s, with controversy surrounding the utility of performing a 'blind' biopsy. ${ }^{7-12}$ This study was performed to compare the costs of conjunctival biopsy with biopsy of other tissue sites in patients eventually confirmed as having systemic sarcoidosis.

\section{Patients and methods}

A retrospective chart review was performed for all patients who had conjunctival biopsy performed at the Mayo Clinic between 1982 and 1995 with the suspected clinical diagnosis of sarcoidosis. Forty-one patients undergoing conjunctival biopsy were diagnosed with sarcoidosis on the basis of a positive tissue biopsy at any site.

Each conjunctival biopsy was performed following a complete ophthalmological evaluation. The biopsy sample size averaged $5 \times 3 \times 2 \mathrm{~mm}$ and was directed towards a suspicious lesion, if any (Table 1). Topical anaesthetic and subconjunctival infiltration of local anaesthetic into the inferior palpebral conjunctiva was employed. The lower lid was retracted and a strip of the ballooned conjunctiva was excised using Westcott scissors. Local pressure and irrigation with Dacriose solution provided adequate haemostasis. Topical antibiotic was then placed in the inferior fornix. There were no complications or morbidity associated with the procedure.
J.A. Leavitt

R.J. Campbell Department of Ophthalmology Mayo Clinic Rochester Minnesota, USA

\section{R.J. Campbell}

Department of Pathology Mayo Clinic

Rochester

Minnesota, USA

Jacqueline A. Leavitt, MD 200 First Street, S.W.

Mayo Clinic

Rochester

MN 55905, USA

Tel: +1 (507) 2843726

Fax: +1 (507) 2844612

e-mail:

leavitt.jacqueline@mayo.edu

Supported in part by an unrestricted grant from Research to Prevent Blindness, Inc., New York, New York 
Table 1. Conjunctival appearance prior to biopsy

\begin{tabular}{lrrrrr}
\hline \multicolumn{3}{l}{ Positive biopsy } & \multicolumn{3}{l}{ Negative biopsy } \\
\hline Nodules & $5 / 21$ & $(24 \%)$ & Nodules & $4 / 20$ & $(20 \%)$ \\
Follicles & $3 / 21$ & $(14 \%)$ & Post-operative bleb & $1 / 20$ & $(5 \%)$ \\
Cyst & $1 / 21$ & $(5 \%)$ & Eyelid mass & $1 / 20$ & $(5 \%)$ \\
No lesions & $12 / 21$ & $(57 \%)$ & No lesions & $14 / 20$ & $(70 \%)$ \\
\hline
\end{tabular}

A thin slice of prepared cucumber ${ }^{13}$ was treated with a drop of glycerol, air dried and used as a flat mount for the biopsy. The tissue on the cucumber was fixed in $10 \%$ buffered formalin and processed in the conventional manner. Paraffin-embedded tissue was sectioned at $5 \mu \mathrm{m}$ at multiple levels (16-20) and stained with haematoxylin and eosin (H\&E). Two additional unstained sections were subsequently stained with Ziehl-Neelsen stain (for acid fast organisms) and silver methenamine stain (for fungi) if granulomas were found on $\mathrm{H} \& \mathrm{E}$-stained material.

\section{Results}

The diagnosis of sarcoidosis was confirmed on tissue biopsy of any site in 41 patients; of these, the conjunctival biopsy was positive in 21 patients (51\%).

Nine nodular conjunctival lesions were subjected to biopsy: 5 were positive for non-caseating granuloma, 1 specimen was a concretion and 3 showed no pathological changes. Chronic non-specific inflammation was found in 6 specimens.

The numbers of non-caseating granulomas in the subepithelial conjunctival tissues ranged from 2 to 6 , but were not present at all levels of the section. Stains for acid fast organisms and fungi were negative. Bilateral conjunctival specimens were obtained in 14 of 21 positive biopsies and 17 of 20 negative biopsies. Of the positive biopsy specimens obtained bilaterally, the majority (71\%) were only positive unilaterally (Table 2 ).

\section{Discussion}

Many previous studies have examined the usefulness of performing conjunctival biopsy in presumed sarcoidosis, with the percentage of positive biopsy ranging from $27 \%$ to $75 \% .^{5-12}$ The first paper exploring the usefulness of conjunctival biopsy was published in 1955 by Crick et al. ${ }^{7}$ They performed 28 conjunctival biopsies. Ten patients had known generalised sarcoidosis and eye involvement; 18 patients presented with uveitis or episcleritis suggestive of sarcoidosis. The conjunctival biopsy was

Table 2. Positive and negative conjunctival biopsies

\begin{tabular}{lrr}
\hline Positive conjunctival biopsy $\left(21\right.$ cases $\left.^{a}\right)$ & & \\
Positive bilaterally & $4 / 14$ & $(29 \%)$ \\
Positive unilaterally (bilateral biopsy) & $10 / 14$ & $(71 \%)$ \\
Positive unilaterally (unilateral biopsy) & $7 / 7$ & $(100 \%)$ \\
Negative conjunctival biopsy (20 cases) & & \\
Bilateral biopsy & $14 / 20$ & $(70 \%)$ \\
Unilateral biopsy & $6 / 20$ & $(30 \%)$ \\
\hline
\end{tabular}

${ }^{a}$ Fourteen bilateral biopsies, seven unilateral biopsies. positive in $30 \%$ and $38 \%$ of cases, respectively. Solomon et al. ${ }^{12}$ found conjunctival 'lesions' in 15 of 21 patients with suspected sarcoidosis studied prospectively. Noncaseating granulomas were found in $8(53 \%)$ of their cases with 'lesions'. All positive biopsies came from African-Americans, so they concluded that patients of this ethnic group were more likely to have sarcoid granulomas in the conjunctiva. Our study included 6 African-Americans and 1 Asian, of whom 4 AfricanAmericans (66\%) and the 1 Asian patient (100\%) had a positive conjunctival biopsy. (In 2 cases the patient's race was not identifiable from the history.)

Comparing conjunctival biopsy with transbronchial biopsy, Spaide and Ward ${ }^{11}$ prospectively studied 47 patients with clinically suspected sarcoidosis. Thirty-four of their patients $(72 \%)$ were found to have

histopathological evidence for sarcoidosis, $40 \%$ were positive on conjunctival biopsy and $66 \%$ on transbronchial biopsy. The conjunctival biopsy was more likely to be positive in the setting of conjunctival follicles $(67 \%)$ than with a normal conjunctiva $(31 \%)$. It was also more often positive in the setting of any ocular abnormality consistent with sarcoidosis (60\% vs $26 \%$ ). Eighteen of our patients (41\%) (10 conjunctival biopsy positive $(55 \%), 8$ biopsy negative $(44 \%))$ had current or prior ocular involvement consistent with sarcoidosis.

Karcioglu and Brear ${ }^{10}$ studied 28 patients prospectively with conjunctival biopsy. Seventy-one per cent of the patients with known sarcoidosis had a positive biopsy whereas $29 \%$ of the suspected cases had a positive result. None of their patients had the classic 'millet-seed' nodules in the conjunctiva and the biopsy site was not directed. Our cases had a number of different non-specific conjunctival 'lesions' (Table 1). Only 8 patients $(19 \%)$ had classic sarcoid nodules (one 'nodule' was pathologically a concretion).

Nichols et al. ${ }^{9}$ found $55 \%$ positive 'blind' conjunctival biopsies in patients with the suspected diagnosis of sarcoidosis. In their study any patient with 'millet-seed' conjunctival nodules was deliberately excluded. The diagnosis of sarcoidosis in all their patients was confirmed with biopsy of another tissue. A key point they made is that conjunctival biopsy should be performed whenever possible on both eyes, since most granulomas were only found unilaterally. Our results concur, in that non-caseating granulomas were found only unilaterally in $71 \%$ of those cases in which bilateral biopsy was performed (Table 2).

Other tissue sites (transbronchial lung biopsy, bronchoalveolar lavage, mediastinoscopy, node biopsy, etc.) are seldom $100 \%$ positive for confirmation of sarcoidosis. A positive tissue biopsy and the stage of sarcoidosis were correlated by Poe et al. ${ }^{14}$ who found a $96 \%$ positive transbronchial biopsy when lung parenchyma was involved versus a $44 \%$ yield without lung involvement. Ohara et al. ${ }^{15}$ studied transbronchial biopsy in patients with suspected ocular sarcoidosis and found this method to be helpful in $62 \%$ of the 60 patients they studied. None of their patients had hilar lymphadenopathy or pulmonary lesions on the chest 
Table 3. Non-ocular biopsy sites to confirm the diagnosis of sarcoidosis

\begin{tabular}{ll}
\hline Positive conjunctival biopsy & Negative conjunctival biopsy \\
\hline Skin/muscle $+7 / 8(87 \%)$ & Bronchoscopy $+6 / 10(60 \%)$ \\
Mediastinoscopy $+3 / 3(100 \%)$ & Mediastinoscopy $+5 / 5(100 \%)$ \\
Lymph node $+3 / 3(100 \%)$ & Lung $+2 / 2(100 \%)$ \\
Liver $+1 / 2(50 \%)$ & Lymph node $+2 / 2(100 \%)$ \\
Bone marrow $+1 / 1(100 \%)$ & Vitreous $+0 / 1$ \\
Lacrimal gland $+1 / 1(100 \%)$ & Brain $+2 / 2(100 \%)$ \\
Thyroid $+1 / 1(100 \%)$ & Spinal cord $+2 / 2(100 \%)$ \\
& Skin $+0 / 2$ \\
& Prostate $+1 / 2(50 \%)$ \\
\hline
\end{tabular}

roentgenogram. After the transbronchial biopsy, one of their patients had a segmental pneumothorax that healed spontaneously in 3 days. In contrast, conjunctival biopsy is a procedure with minimal risks, and none of the patients in our study had any complications.

Gilman and Wang ${ }^{16}$ found the probability of a positive transbronchial biopsy in stage II sarcoidosis to be $46 \%$ per biopsy specimen. Their diagnostic yield increased logarithmically such that the probability of a positive biopsy increased to $90 \%$ after four biopsy specimens. Takayama et al. ${ }^{17}$ found that step-sectioning (serial sections in transbronchial specimens) increased the positive yield on transbronchial biopsy from $38 \%$ to $47 \%$ in patients with stage I and disease and from $57 \%$ to $82 \%$ in stage II. Serial sectioning of the conjunctiva specimen should also be examined. One of our positive cases was initially read as negative, but when reviewed for the purposes of this study was found on additional sectioning to be positive.

Our 21 patients with a positive conjunctival biopsy underwent 19 additional tissue biopsies ( $90 \%$ positive), some pre-dating the conjunctival biopsy and some performed at other institutions. The 20 patients with negative conjunctival biopsies underwent an additional 28 biopsies (71\% positive) (Table 3 ). Costs for the conjunctival biopsy performed in the outpatient setting were less expensive than any of the other biopsy procedures (Table 4 ). In fact 10 conjunctival biopsies could be performed for the same cost as one mediastinoscopy. The patients with a positive conjunctival biopsy could have been saved 17 procedures at an estimated cost of $\$ 81350$ to $\$ 1121150$ in 1997 dollars. Since our study found $51 \%$ of the patients with pathologically proven sarcoidosis had a positive conjunctival biopsy, this procedure could be performed on anyone meeting the clinical and laboratory criteria for sarcoidosis to spare the additional expense of the more invasive, potentially more complicated biopsies.

In summary, pathological confirmation of sarcoidosis is similar following either conjunctival biopsy or mediastinal biopsy, with both procedures more likely to be positive in more advanced disease states and with increased tissue sampling. The conjunctival biopsy may be positive in approximately $50 \%$ of patients with sarcoidosis. The most helpful conjunctival specimen for biopsy purposes is a strip of tissue $8-10 \mathrm{~mm}$ long from the inferior conjunctival fornix. The pathologist must step-section the conjunctival specimens as the non-
Table 4. Cost estimates ${ }^{a}$ for tissue biopsies

\begin{tabular}{lc}
\hline Site & Cost (\$US, 1997) \\
\hline Bilateral conjunctival biopsy & $590-930$ \\
Mediastinoscopy & $6800-9300$ \\
Lacrimal gland biopsy & $900-1350$ \\
Bone marrow biopsy & $1050-1650$ \\
Skin/muscle biopsy & $3450-5400$ \\
Lymph node biopsy & $5100-6150$ \\
Percutaneous liver biopsy & $3500-4200$ \\
Thyroidectomy & $9100-11200$ \\
Bronchoscopy with BAL & $2900-4100$ \\
Bronchoscopy with TBB & $3300-4450$ \\
Brain biopsy via burr hole & $10600-14900$ \\
Spinal cord biopsy & $5800-7300$ \\
Scalene node biopsy & $4900-7400$ \\
Vitreous biopsy & $6800-8600$ \\
Prostate biopsy & $2800-3800$ \\
\hline BAL, bron
\end{tabular}

$\mathrm{BAL}$, bronchoalveolar lavage; TBB, transbronchial biopsy. ancludes the procedure, surgeon, operating room, anaesthesia, pathology, post-operative recovery and miscellaneous fees at Mayo Clinic Rochester, all given in 1997 dollar figures. Several procedures were not performed at the Mayo Clinic and predated the ultimate diagnosis of sarcoidosis. All conjunctival biopsies were performed at Mayo Clinic Rochester.

caseating granulomas are not uniformly distributed. Of all the biopsy techniques performed to confirm sarcoidosis, conjunctival biopsy is the least expensive and least complicated. Compared with mediastinoscopy, conjunctival biopsy is one-tenth the cost. Performance of a conjunctival biopsy may save the patient a more costly and complex biopsy procedure while securing the diagnosis of sarcoidosis.

\section{References}

1. Weinreb RN, Tessler H. Laboratory diagnosis of ophthalmic sarcoidosis. Surv Ophthalmol 1984;28:653-64.

2. Bienfait MF, Hoogsteden HC, Baarsma GS, Adriaansen HJ, Verheijen-Breemhaar L. Diagnostic value of bronchoalveolar lavage in ocular sarcoidosis. Acta Ophthalmol (Copenh) 1987;65:745-8.

3. James DG, Neville E, Langley DA. Ocular sarcoidosis. Trans Ophthalmol Soc UK 1976;96:133-9.

4. Dresner MS, Brecher R, Henkind P. Ophthalmology consultation in the diagnosis and treatment of sarcoidosis. Arch Intern Med 1986;146:301-4.

5. Collison JMT, Miller NR, Green WR. Involvement of orbital tissue by sarcoid. Am J Ophthalmol 1986;102:302-7.

6. Smith JA, Foster CS. Sarcoidosis and its ocular manifestations. Int Ophthalmol Clin 1996;36:109-25.

7. Crick R, Hoyle C, Mather G. Conjunctival biopsy in sarcoidosis. BMJ 1955;2:1180-1.

8. Merritt JC, Lipper SL, Peiffer RL, Hale LM. Conjunctival biopsy in sarcoidosis. J Natl Med Assoc 1980;72:347-9.

9. Nichols DW, Eagle RC Jr, Yanoff M, Menocal NG. Conjunctival biopsy as an aid in the evaluation of the patient with suspected sarcoidosis. Ophthalmology 1980;87:287-91.

10. Karcioglu AZ, Brear R. Conjunctival biopsy in sarcoidosis. Am J Ophthalmol 1985;99:68-73.

11. Spaide RF, Ward DL. Conjunctival biopsy in the diagnosis of sarcoidosis. Br J Ophthalmol 1990;74:469-71.

12. Solomon DA, Horn BR, Byrd RB, Lorfel RS, Griggs GA. The diagnosis of sarcoidosis by conjunctival biopsy. Chest 1987;74:271-3.

13. Erie JC, Collyer SK, Campbell RJ. Dehydrated cucumber slice as a mount for conjunctival biopsy specimens. Am J Ophthalmol 1985;99:539-41. 
14. Poe RH, Israel RH, Utell MJ, Hall WJ. Probability of a positive transbronchial lung biopsy result in sarcoidosis. Arch Intern Med 1979;139:761-3.

15. Ohara K, Okubo A, Kamata K, Sasaki H, Kobayashi J, Kitamura S. Transbronchial lung biopsy in the diagnosis of suspected ocular sarcoidosis. Arch Ophthalmol 1993;111:642-4.
16. Gilman MJ, Wang KP. Transbronchial lung biopsy in sarcoidosis. Am Rev Resp Dis 1980;122:721-4.

17. Takayama K, Nagata N, Miyagawa Y, Hirano H, Shigematsu $\mathrm{N}$. The usefulness of step sectioning of transbronchial lung biopsy specimen in diagnosing sarcoidosis. Chest 1992;102:1441-3. 